\title{
Caracterização Morfológica do Poliestireno de Alto Impacto (HIPS)
}

\author{
Juliana Rovere, Carlos A. Correa \\ Laboratório de Blendas e Compósitos Termoplásticos, USF
}

\author{
Vinícius G. Grassi, Marcus F. Dal Pizzol \\ Innova S.A.
}

Resumo: Poliestireno de alto impacto (HIPS) é uma blenda polimérica clássica de reator produzida por polimerização in-situ de estireno em solução com borracha butadiênica. Na literatura, está bem estabelecida a importância do tamanho de partícula e da densidade da ligação cruzada da borracha na capacidade de cavitação e no controle dos mecanismos de tenacificação na matriz estirênica. No presente trabalho as técnicas de microscopia eletrônica de transmissão (MET) e espalhamento de luz laser de baixo ângulo (LALLS) foram empregadas para observar a distribuição do tamanho de partícula, e a análise digital de imagem foi aplicada para quantificar os parâmetros morfológicos nas micrografias. Medidas do tamanho de partícula e da fração aparente volumétrica de partícula foram feitas para diferentes espessuras na MET e comparadas com o parâmetro estatístico $\mathrm{D}^{[4,3]}$ obtido na LALLS.

Palavras-chave: HIPS, morfologia, conteúdo cis, tenacidade.

\section{Morphological Characterization of High Impact Polystyrene (HIPS)}

Abstract: High impact polystyrene (HIPS) is a classical reactor polymer blend produced by in-situ polymerization of styrene in solution with polybutadiene rubber. It is well established in the literature the importance of particle size and rubber crosslink density on the particle cavitation capability and on the controlling of toughening mechanisms in the styrene matrix. In the present work transmission electron microscopy (TEM) and low-angle laser light scattering (LALLS) were employed for investigating particle size distribution, with digital imaging being applied in the quantitative analysis of the micrographs. Measurements of apparent volume fraction and average particle size were carried out for different slice thicknesses in TEM and compared with the statistical parameter $\mathrm{D}^{[4,3]}$ obtained by LALLS.

Keywords: HIPS, morphology, cis-content, toughness.

\section{Introdução}

Muitos polímeros amorfos ou vítreos tendem a apresentar comportamento frágil à fratura limitando assim sua gama de aplicações. O reforçamento com elastômeros é reconhecidamente a técnica mais utilizada por grande parte das indústrias de polímeros para aumentar a resistência à fratura e a tenacidade desses materiais ${ }^{[1,2]}$. Nesta importante classe de compósitos poliméricos encontra-se o Poliestireno de Alto Impacto (PSAI ou high impact polystyrene - HIPS) que é tipicamente caracterizado por uma segunda fase borrachosa (polibutadieno - PB) dispersa em uma matriz polimérica contínua (poliestireno - PS) ${ }^{[3]}$. Esta classe é conhecida como um típico material polimérico borrachoso tenacificado preparado pela polimerização de um radical livre de estireno na presença de polibutadieno dissolvido, aumentando a resistência ao impacto e a tenacidade do poliestireno vítreo ${ }^{[4]}$.
O HIPS é um material com ampla aplicação. Em artigos industriais é usado como peças internas e externas de aparelhos eletrônicos, de telecomunicações, toca-fitas de carro, cabos e armações para guarda-chuvas, gabinetes para geladeiras, peças de máquinas e veículos, caixas para rádio, televisão, microcomputadores e grades de ar condicionado; também é utilizado nas indústrias de calçados (saltos) e de embalagens de proteção contra choques ${ }^{[5]}$. Pode ser empregado também em diversos utensílios, como brinquedos, jogos, artigos descartáveis e utilidades domésticas ${ }^{[6]}$.

Como o HIPS é um material polimérico composto de múltiplos componentes e múltiplas camadas, com fases vítreas e borrachosas, o bom desempenho do material modificado depende de diversos parâmetros ${ }^{[7,8]}$. Um parâmetro relacionado à fase vítrea (matriz) seria a massa molecular, pois ela influencia no tipo de processamento, na forma como as cadeias estão organizadas (em faixas estreitas ou largas)

Autor para correspondência: Carlos A. Correa, Laboratório de Blendas e Compósitos Termoplásticos, Universidade São Francisco, Rua Alexandre Rodrigues Barbosa 45, CEP: 13251-900, Itatiba, SP, Brasil. E-mail: carlos.correa@saofrancisco.edu.br 
e na viscosidade do polímero - resultando em polímeros com características distintas. Vários parâmetros relacionados à fase borracha têm sido identificados como modificadores da tenacidade deste sistema, como: fração volumétrica da fase borracha (este parâmetro é influenciado pelo conteúdo de borracha, pelo número e tamanho das oclusões e pelo grau de graftização), densidade de ligação cruzada (o grau de entrecruzamento determina a cavitação da borracha), morfologia da partícula, nível de adesão com a matriz, tipo de borracha (na maioria dos casos o tipo de borracha é o polibutadieno (alto e baixo cis), porém outros tipos de borracha ocasionalmente utilizados são o poli(butadieno-co-estireno) (SBR) e o poli(etileno-co-propileno-co-dieno) (EPDM)) e, os mais importantes, tamanho e distribuição de partículas de borracha, pois definem algumas características de acabamento superficial e transparência destes materiais, além de ser crítico para as propriedades mecânicas ${ }^{[9]}$.

A escolha do polibutadieno é determinada pelas características finais a serem atingidas no HIPS, sendo as principais a viscosidade - decorrente do peso molecular e do grau de ramificações - e a configuração molecular (isomeria) no que se refere às porcentagens relativas das insaturações nas configurações vinil, trans e cis, como representado na Figura $1^{[10,11]}$.

A caracterização do polibutadieno no HIPS pode ser realizada através da determinação da razão fase-volume ou teor de gel, que por sua vez representa a fração insolúvel do HIPS no tolueno, segundo a técnica de separação de fases de Ruffing ${ }^{[12]}$. A fase gel no HIPS é composta pelo PB não graftizado (homopolímero), pelo PB entrecruzado, pelo copolímero graftizado PS-PB e pelo PS ocluído no interior das partículas de borracha. As cadeias de copolímero graftizado PS-PB ficam dispostas na interface entre a partícula de borracha e a matriz de PS, magnificando a adesão entre ambas ${ }^{[13]}$. Quanto maior a quantidade de PS aprisionado nas partículas após a inversão de fase, maior é a razão fase-volume PB/PS. No HIPS comercial utiliza-se de 6-10\% em massa de borracha; de $25-35 \%$ de conteúdo de gel e de $75-80 \%$ de oclusões na partícula ${ }^{[9]}$.

Nos processos industriais para produção de polibutadieno em solução, utiliza-se a catálise de coordenação, mais especificamente complexos com metais de transição, como cobalto (PB-Co) e neodímio (PB-Nd) ${ }^{[14]}$. Estes processos geram os chamados polibutadienos de alto-cis, nos quais o conteúdo do isômero 1,4-cis varia entre 96 e 98\% ${ }^{[10]}$. Métodos como Espectroscopia no Infravermelho com Transformada de Fourier (FTIR), Ressonância Magnética Nuclear (RMN) e titulome-

$$
\left[\begin{array}{r}
\mathrm{CH}_{2}-\mathrm{CH} \\
\mathrm{I} \\
\mathrm{CH} \\
\stackrel{\mathrm{I}}{\mathrm{C}} \mathrm{H}_{2}
\end{array}\right]_{\mathrm{n}}
$$

(a)

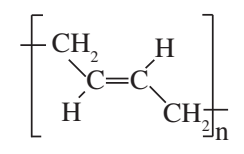

(b)

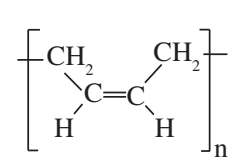

(c)
Figura 1. Representação das insaturações; a) 1,2-vinil; b) 1,4-trans; e c) 1,4-cis, em diferentes configurações nas moléculas de PB. tria, são utilizados para quantificação do teor do isômero cis em polibutadieno ${ }^{[15-19]}$. Os polibutadienos de alto-cis tendem a gerar HIPS com menor grau de entrecruzamento na fase borracha, favorecendo a resistência ao impacto e a ductilidade. Por outro lado, tendem a gerar menor nível de graftização, o que pode se refletir negativamente sobre a morfologia e, até mesmo, comprometer as propriedades mecânicas ${ }^{[20]}$. No entanto, a escolha do polibutadieno não afeta diretamente as características morfológicas do material, apenas as propriedades mecânicas.

As diferenças estruturais e morfológicas entre os tipos de HIPS resultam de diferenças nos processos de produção e no tipo e teor de borracha ${ }^{[21,22]}$. O processo de polimerização em massa gera morfologia do tipo salami, com espectro amplo de tamanho de partículas, com subinclusões de PS envoltos por uma membrana borrachosa, dispersa numa matriz de PS. O processo de polimerização em emulsão geralmente apresenta partículas de borracha esféricas, não-desejáveis por comprometer a transparência, com estrutura do tipo core-shell, isto é, partículas compostas por um núcleo de PS (core) revestido por uma membrana (shell), com espectro de distribuição de tamanhos mais estreito ${ }^{[23]}$.

Existe uma infinidade de técnicas experimentais que fornecem informações a diferentes níveis sobre a microestrutura do material, destacando-se as microscopias óptica (MO) ou eletrônica (ME), análise química, calorimetria diferencial de varredura (DSC) e técnicas de espalhamento de luz (espalhamento de luz laser de baixo ângulo - LALLS). A princípio, as blendas polímero-borracha podem ser caracterizadas somente pelo peso de cada componente da blenda ou pelo método do índice de inchamento. A utilização tanto da microscopia eletrônica de varredura (MEV) quanto da de transmissão (MET) e da técnica de espalhamento de luz para a caracterização dos polímeros modificados com borracha possui particular interesse por ser uma técnica mais direta ${ }^{[24]}$.

Na análise por MET, a preparação da amostra é uma parte crítica e trabalhosa da análise; é necessário o uso de um ultramicrótomo para preparar fatias na faixa de 70 a $100 \mathrm{~nm}$. Devido à baixa variação de densidade eletrônica entre os constituintes das misturas polímero-borracha, utiliza-se a técnica de manchamento com o tetraóxido de ósmio $\left(\mathrm{OsO}_{4}\right)$ para se obter melhor contraste de imagem entre ambas as fases. $\mathrm{O} \mathrm{OsO}_{4}$ reage preferencialmente com as duplas ligações da borracha insaturada por reação de graftização, aumentando a diferença de densidade eletrônica entre as duas fases. $\mathrm{O}$ aumento da intensidade de espalhamento de elétrons nas regiões ricas em borracha comparadas à matriz não manchada produz um escurecimento dessas regiões na imagem. Avanços na análise digital de imagem permitem quantificar medidas de aspectos morfológicos em imagens com o auxílio de técnicas computacionais. Um analisador de imagens típico processa informação quantitativa em termos de número, tamanho, área, forma e outros aspectos detectáveis da imagem. Desta forma, a resolução da imagem está diretamente relacionada com o 
número de pixels e a capacidade do software computacional empregado na manipulação dos dados ${ }^{[24]}$.

$\mathrm{Na}$ análise por LALLS, as partículas passam através de um feixe de radiação de luz (atualmente, todos os detectores utilizam o laser como fonte de luz, por exemplo, $\mathrm{He} / \mathrm{Ne}^{[25]}$ ), provocando o espalhamento da luz em um ângulo que é inversamente proporcional ao seu tamanho. A luz espalhada é coletada por um detector e analisada com base em um padrão de difração, definido antecipadamente ${ }^{[26]}$. $\mathrm{O}$ padrão de um grupo de partículas é idêntico à soma dos padrões individuais de espalhamento de luz de todas as partículas presentes ${ }^{[27]}$. Como resultado da análise, pode-se obter uma curva de distribuição de tamanhos de partícula da borracha gerada com base na contribuição da frequiência de cada fração $0^{[10]}$.

O objetivo deste trabalho é empregar as técnicas de MET e LALLS para observar e comparar a distribuição do tamanho de partícula de borracha em formulações comerciais de HIPS. Análise morfológica quantitativa foi empregada para quantificar o espectro de tamanho de partícula e as respectivas frações volumétricas. Estas medidas foram efetuadas através da análise por MET, com diferentes espessuras, e foram comparadas com o parâmetro estatístico $\mathrm{D}^{[4,3]}$ obtido por LALLS.

\section{Experimental}

\section{Materiais}

Neste trabalho foram utilizados dois tipos de HIPS comerciais aqui denominados HIPS 1 e HIPS 2, fornecidos pela Innova S.A. Esses materiais foram escolhidos com base nas diferenças no tamanho de partícula de borracha médio e na distribuição. A morfologia interna das partículas é a mesma a tradicional morfologia do tipo salami. Um resumo dos principais parâmetros moleculares e estruturais medidos por métodos de caracterização (descritos nas notas da tabela) para os materiais puros encontram-se apresentados na Tabela 1.

\section{Métodos}

Os materiais foram moldados por compressão a $195{ }^{\circ} \mathrm{C}$ em placas de $4 \mathrm{~mm}$ de espessura, e cargas de 150 a $1000 \mathrm{kgf}$ (Tabela 2). Após a moldagem, as chapas foram resfriadas até $30{ }^{\circ} \mathrm{C}$. O equipamento utilizado para a moldagem das placas por compressão foi um Mecanoplast, modelo PLR 25. As espécies para os ensaios mecânicos foram cortadas a partir destas placas.

\section{Análise morfológica e análise de imagem}

A análise de imagem por MET foi usada para caracterizar as formulações selecionadas em termos de sua fração aparente de partícula, $\Phi_{\mathrm{A}}$, e seu diâmetro equivalente de partícula, $\mathrm{D}_{\text {eq }}$ O número mínimo de partículas que deve ser utilizado para calcular o diâmetro médio de partícula depende da amostra, como pode ser observado na literatura ${ }^{[28]}$. Foram
Tabela 1. Parâmetros gerais das amostras de HIPS estudadas.

\begin{tabular}{|c|c|c|c|}
\hline \multirow[t]{2}{*}{ Propriedades } & \multirow[t]{2}{*}{ Unidade } & \multicolumn{2}{|c|}{ Material } \\
\hline & & HIPS 1 & HIPS 2 \\
\hline $\mathrm{Mw} \times 10^{-3(\mathrm{a})}$ & $\mathrm{g} / \mathrm{mol}$ & 172 & 185 \\
\hline $\operatorname{Mn} \times 10^{-3(\mathrm{~b})}$ & $\mathrm{g} / \mathrm{mol}$ & 239 & 270 \\
\hline $\mathrm{Mw} / \mathrm{Mn}^{(\mathrm{c})}$ & - & 1,9 & 1,88 \\
\hline Teor de gel $^{(\mathrm{d})}$ & $\%$ & 18,1 & 11,8 \\
\hline Índice de inchamento ${ }^{(\mathrm{e})}$ & - & 11,8 & 24,5 \\
\hline Óleo mineral ${ }^{(\mathrm{f})}$ & $\%$ & 0,6 & 1,0 \\
\hline $\mathrm{PB}\left({ }^{*(g)}\right.$ e $\left.\#^{(\mathrm{h})}\right)$ & $\%$ & $5.1 \#$ & $5.5^{*}$ \\
\hline Conteúdo Cis-PB ${ }^{(\mathrm{i})}$ & - & baixo & alto \\
\hline
\end{tabular}

(a) Mw: massa molecular ponderal media do PS (g/mol) determinada por cromatografia por permeação a gel (G.P.C.); (b)Mn: massa molecular numérica média do PS (g/mol); ( ${ }^{(\mathrm{M}} \mathrm{Mw} / \mathrm{Mn}$ : distribuição da massa molecular; ${ }^{(\mathrm{d})}$ Teor de gel: fração em peso da segunda fase (\%) medida por métodos de separação de fase em uma solução selecionada (as amostras foram dissolvidas e lavadas em metil etil cetona (MEK) e solução de tolueno (proporção 43:57 v/v), a parte insolúvel foi separada por centrifugação, depois foi precipitada por etanol, centrifugada e então filtrada a vácuo, colocada na estufa e depois em um dessecador e por fim, foi pesada); ${ }^{(e)}$ índice de inchamento: razão entre o gel inchado e seco (\%). O gel seco é medido pelo método de separação de fase em um solvente seletivo (tolueno); o índice de inchamento das amostras em tolueno é dado pela proporção entre gel inchado e seco; ${ }^{(\mathrm{f})}$ óleo mineral: medido pelo método de Espectroscopia no Infravermelho com Transformada de Fourier (FTIR); ${ }^{(\mathrm{g})} \mathrm{PB} *$ : fração em peso do PB (\%) medido pelo método de titulação; (h)PB\#: fração em peso do PB (\%) medido pelo método de FTIR; ${ }^{(i)}$ conteúdo de cis polybutadiene: medido pelo método de FTIR ou pelo método da espectroscopia de ressonância magnética nuclear (RMN).

Tabela 2. Razões de aquecimento utilizadas na produção das placas.

\begin{tabular}{cccc}
\hline Etapa & Temperatura $\left({ }^{\circ} \mathbf{C}\right)$ & Cargas $(\mathbf{k g})$ & Tempo $(\mathbf{s})$ \\
\hline 1 & 195 & 150 & 15 \\
2 & 195 & 150 & 15 \\
3 & 195 & 600 & 15 \\
4 & 195 & 800 & 0 \\
5 & 195 & 1000 & 50 \\
\hline
\end{tabular}

analisadas cinco micrografias por amostras, independente do número de partículas em cada micrografia. No entanto, como valor recomendado ${ }^{[28]}$ considerou-se uma freqüência acima de 200 partículas. A morfologia dos polímeros por MET foi observada utilizando um microscópio eletrônico Philips, modelo EM208S operado a $80 \mathrm{kV}$. Amostras poliméricas com espessuras de 100, 120 e $150 \mathrm{~nm}$ foram preparadas utilizando um ultramicrótomo Leica, modelo UCT Ultracut. Os softwares utilizados na análise de imagens foram o Jasc Paint Software, da Jasc Software e o JMicro Vision, da Nicolas Roduit.

A análise do tamanho médio de partícula de borracha também foi realizada por meio da técnica LALLS, utilizando o equipamento Mastersizer Micro Particle Analyzer, da Malvern Instruments. O equipamento cobre um intervalo de tamanho de partículas de 0,02 a $2000 \mu \mathrm{m}$. Para a análise, uma amostra de $0,03 \mathrm{~g}$ de gel seco foi dispersa em aproximadamente $25 \mathrm{~mL}$ de metiletilcetona (MEK). A amostra foi mantida durante 20 segundos sob ultra-som para uma melhor dispersão do gel no solvente. A amostra foi adicionada à célula do analisador de tamanho de partícula, à qual já havia 
sido adicionado aproximadamente $150 \mathrm{~mL}$ de etanol (meio dispersante).

\section{Resultados e Discussão}

\section{Caracterização por MET, LALLS e análise morfológica quantitativa}

No processamento, a imagem cinza (Figura 2a) é transformada em uma imagem digital binária (Figura 2b) de forma que as relações entre as regiões claras e escuras possam ser determinadas e processadas através de métodos digitais de tratamento de imagens.

Após o processamento da imagem, os parâmetros de interesse - volume da fração aparente de partícula, $\Phi_{\mathrm{A}}$, e seu diâmetro equivalente de partícula, $\mathrm{D}_{\mathrm{eq}}-$ foram medidos. A $\Phi_{\mathrm{A}}$ é obtida dividindo a área ocupada pelas partículas de borracha $\left(A_{B}\right.$, correspondente às regiões escuras) pela área total da micrografia ( $\mathrm{A}_{\mathrm{T}}$ correspondente ao fundo claro) (Equação 1). O parâmetro $\mathrm{D}_{\text {eq }}$ é calculado de acordo com a Equação 2, em que A é a área da partícula.

$$
\begin{gathered}
\Phi_{A}=\frac{\sum A_{B}}{\sum A_{T}} \\
\mathrm{D}_{e q}=2 \sqrt{\frac{A}{\pi}}
\end{gathered}
$$

O histograma que representa o espectro do tamanho de partícula foi obtido dividindo o espectro em 20 intervalos de $\mathrm{D}_{\text {eq }}$ em função da frequiência observada. Os principais parâmetros utilizados neste processo estão demonstrados nas Equações 3, 4 e 5, mostrando como eles foram calculados.

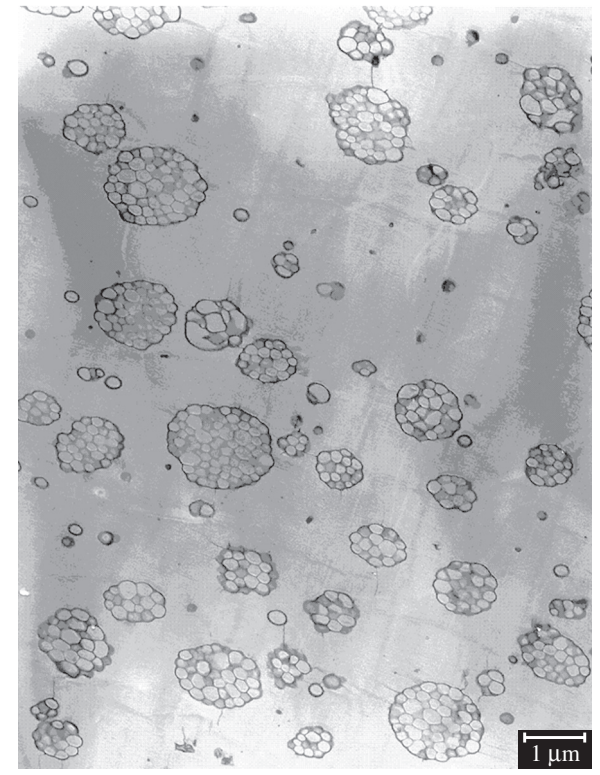

(a)

$$
\begin{aligned}
& D n=\frac{\sum N i D i}{\sum N i} \\
& D w=\frac{\sum N i D i^{2}}{\sum D i N i} \\
& D D_{e q}=\frac{D w}{D n}
\end{aligned}
$$

em que Di $=$ diâmetro médio de partículas dentro do intervalo; $\mathrm{Dn}=$ diâmetro equivalente numérico médio; $\mathrm{Dw}=\mathrm{di}-$ âmetro equivalente ponderal médio; DDeq = distribuição do diâmetro de partícula.

O parâmetro $\mathrm{DD}_{\text {eq }}$ é calculado a partir do conceito estatístico de dispersividade da distribuição da amostra. O parâmetro é uma medida da largura da distribuição de diâmetros de partículas, ou seja, quanto mais afastado $\mathrm{DD}_{\text {eq }}$ estiver da unidade, mais larga a distribuição dos diâmetros e mais heterogênea a amostra.

Na Figura 3a-c está ilustrada a morfologia observada por MET para o HIPS 1, para as espessuras de 100, 120 e $150 \mathrm{~nm}$, respectivamente. Os histogramas correspondentes à distribuição do tamanho de partícula estão representados na Figura 3d-f.

Na Figura 4a-c está ilustrada a morfologia observada por MET para o HIPS 2, para as espessuras de 100, 120 e $150 \mathrm{~nm}$, respectivamente. Os histogramas correspondentes à distribuição do tamanho de partícula estão representados na Figura 4d-f.

De acordo com as Figuras 3d-f e 4d-f) os resultados da análise do tamanho de partícula por MET para diferentes espessuras são demonstrados na Tabela 3 .

Os resultados da análise dos histogramas e da Tabela 3 indicam que o DM para as três espessuras da MET para o

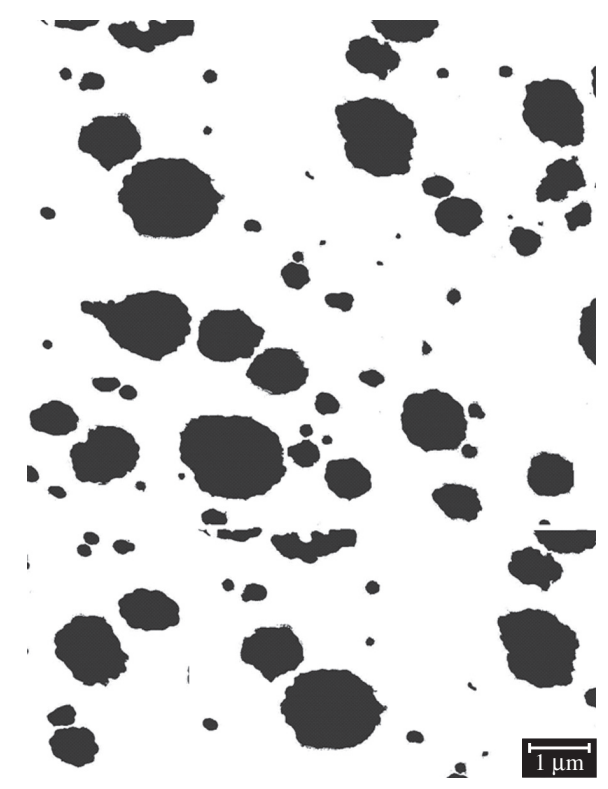

(b)

Figura 2. Imagem obtida do HIPS 1 por a) MET; e b) Forma binária da imagem obtida por processamento digital de imagem. 


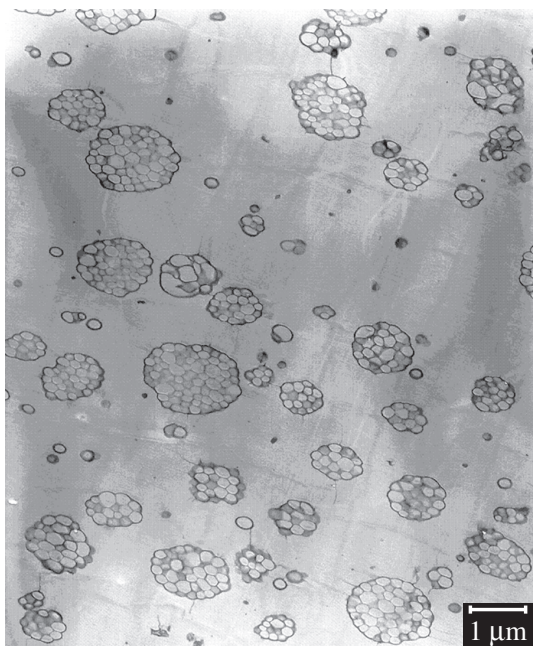

(a)

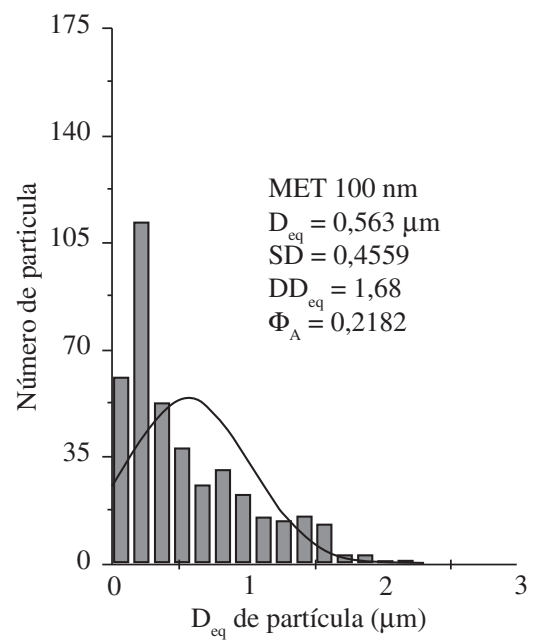

(d)

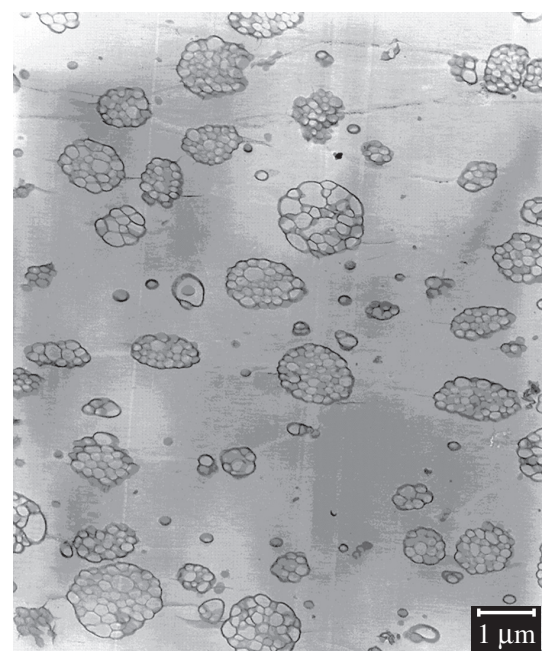

(b)

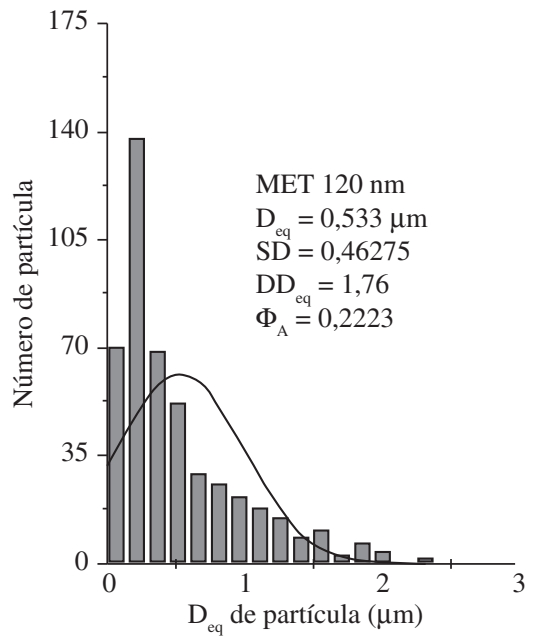

(e)

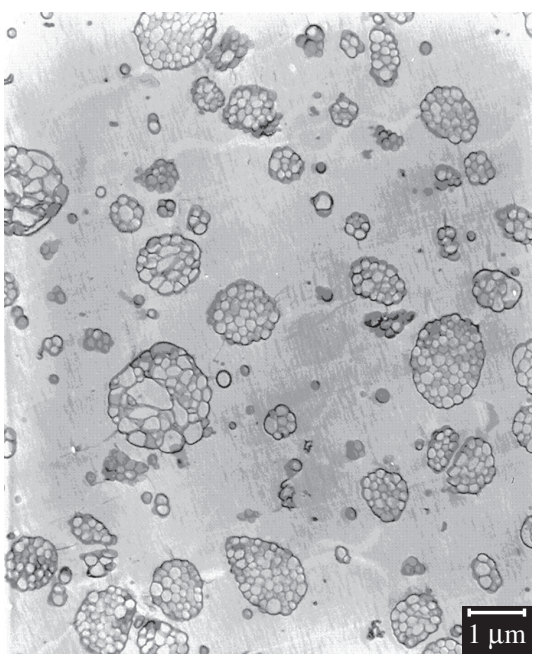

(c)

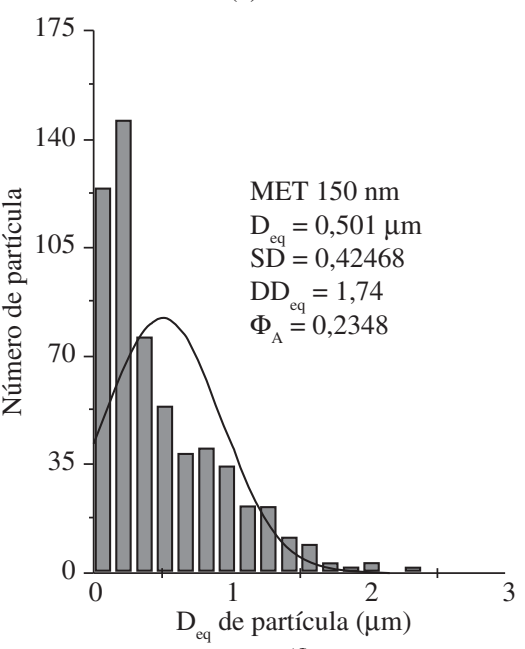

(f)

Figura 3. Espectro do tamanho de partícula para micrografias do HIPS 1, obtida por MET, com espessura de a) $100 \mathrm{~nm}$; b) $120 \mathrm{~nm}$; c) $150 \mathrm{~nm}$ com o respectivo histograma de tamanho de partícula; d) $100 \mathrm{~nm}$; e) $120 \mathrm{~nm}$; e f) $150 \mathrm{~nm}$.

Tabela 3. Resultados da análise de partícula por MET.

\begin{tabular}{|c|c|c|c|c|c|c|c|c|c|c|}
\hline & \multicolumn{2}{|c|}{ Espessura } & \multicolumn{2}{|c|}{ DM $(\mu \mathrm{m})$} & \multicolumn{2}{|c|}{$\mathrm{DD}_{\mathrm{eq}}$} & \multicolumn{2}{|c|}{$\mathbf{N}$} & \multicolumn{2}{|c|}{$\Phi \mathbf{A}$} \\
\hline & HIPS 1 & HIPS 2 & HIPS 1 & HIPS 2 & HIPS 1 & HIPS 2 & HIPS 1 & HIPS 2 & HIPS 1 & HIPS 2 \\
\hline \multirow[t]{3}{*}{ MET } & $100 \mathrm{~nm}$ & $100 \mathrm{~nm}$ & 0,56 & 0,50 & 1,68 & 2,28 & 410 & 430 & 0,21 & 0,22 \\
\hline & $120 \mathrm{~nm}$ & $120 \mathrm{~nm}$ & 0,53 & 0,69 & 1,76 & 2,00 & 474 & 309 & 0,22 & 0,23 \\
\hline & $150 \mathrm{~nm}$ & $150 \mathrm{~nm}$ & 0,50 & 0,72 & 1,74 & 2,01 & 584 & 413 & 0,23 & 0,25 \\
\hline
\end{tabular}

$\mathrm{D}_{\mathrm{M}}=$ Diâmetro médio de partículas no histograma; $\mathrm{N}=$ Número de partículas analisadas; $\mathrm{DD}_{\text {eq }}=$ distribuição do $\mathrm{D}_{\text {eq }} ; \Phi_{\mathrm{A}}=$ volume da fração aparente de partículas de borracha.

HIPS 1 são aproximadamente os mesmos, isto é, a espessura não tem uma grande influência neste parâmetro. No entanto, o HIPS 2 mostra-se levemente dependente da espessura. Analisando os resultados encontrados para a distribuição do diâmetro equivalente de partícula $\left(\mathrm{DD}_{\mathrm{eq}}\right)$ verificou-se que não há dependência deste parâmetro com a espessura. Os valores estão de acordo com a largura da distribuição de tamanho de partículas observados nas Figuras 3 e 4.

$\mathrm{O}$ parâmetro $\mathrm{N}$ seguiu uma freqüência mínima observada acima de 200 partículas, conforme estudos anteriores ${ }^{[28]}$, uma vez que estes parâmetros tendem a se manter constantes a partir de uma determinada freqüência. A fração aparente $\left(\Phi_{\mathrm{A}}\right)$ para os dois materiais é praticamente constante e mostra-se independente da espessura do filme

$\mathrm{Na}$ análise por LALLS, o parâmetro de interesse, diâmetro médio volumétrico de partícula $\left(\mathrm{D}^{[4,3]}\right)$, foi medido. Ele é obtido através do quociente da somatória dos diâmetros elevada a quarta potência pela somatória dos diâmetros elevada a terceira potência (Equação 6).

$$
D^{[4,3]}=\frac{\sum d^{4}}{\sum d^{3}}
$$




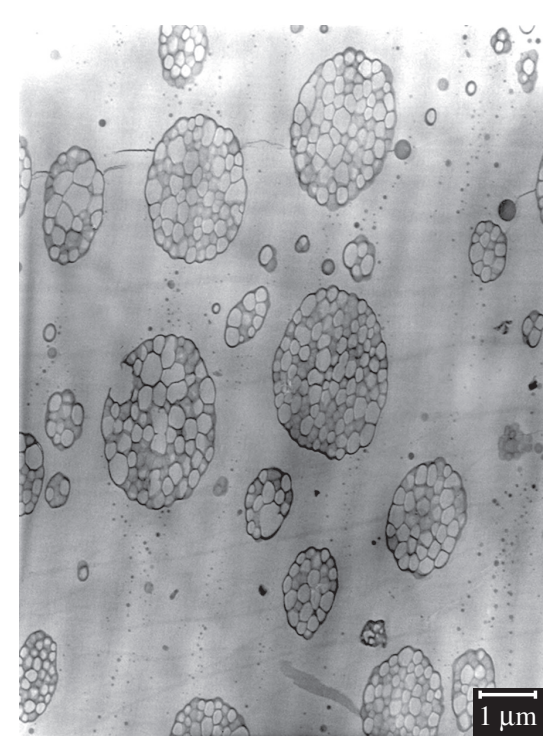

(a)

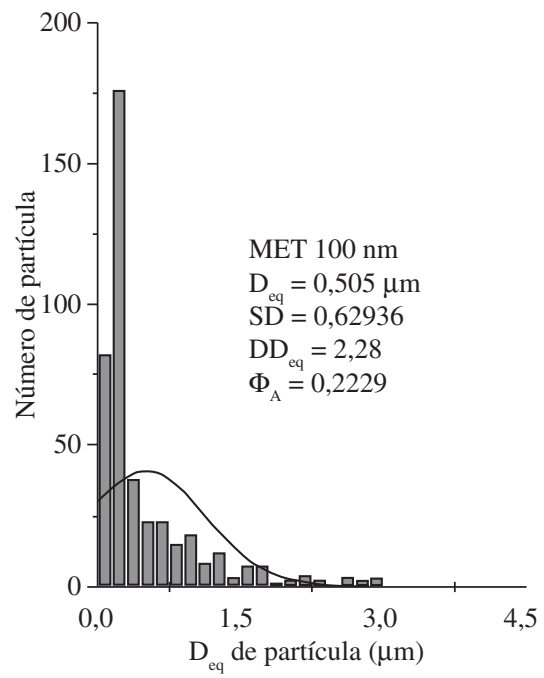

(d)

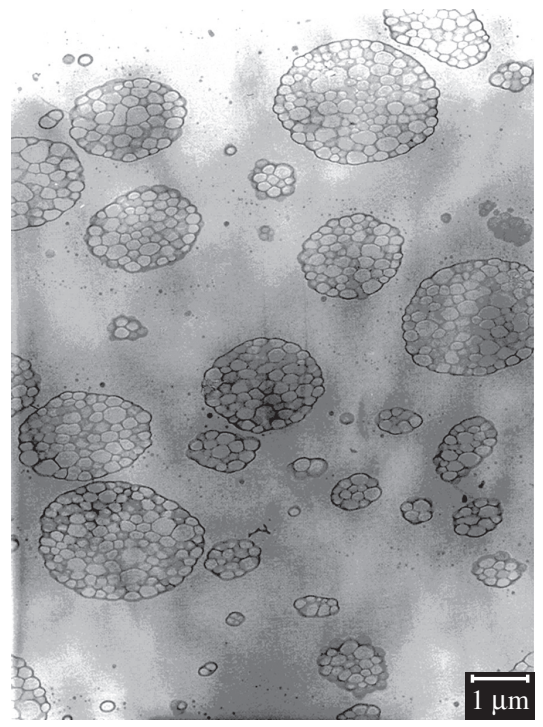

(b)

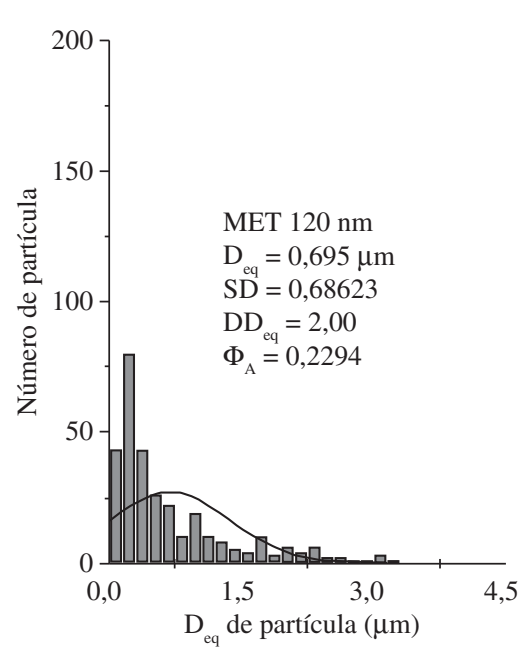

(e)

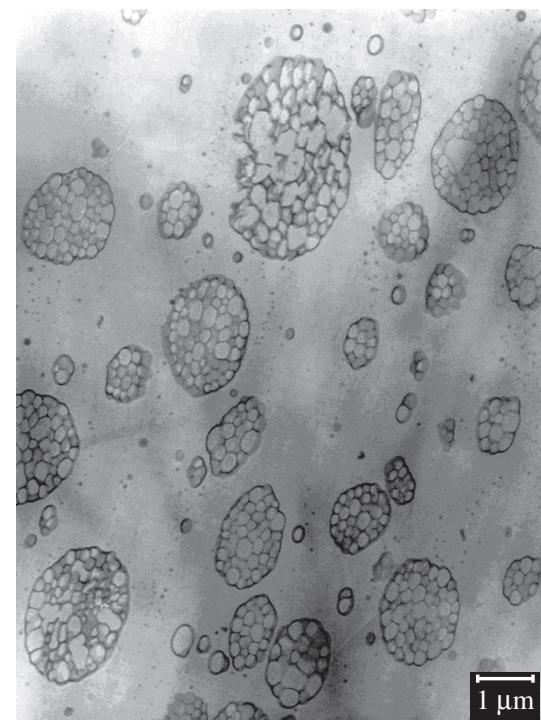

(c)

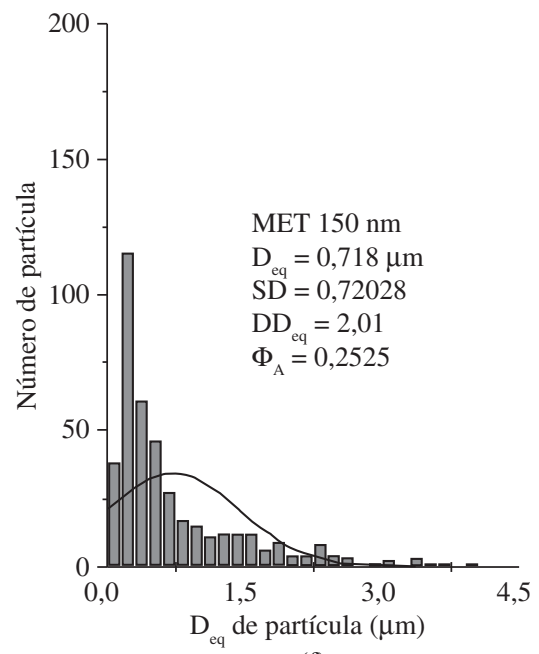

(f)

Figura 4. Espectro do tamanho de partícula para micrografias do HIPS 2, obtida por MET, com espessura de a) $100 \mathrm{~nm}$; b) $120 \mathrm{~nm}$; c) $150 \mathrm{~nm}$ com o respectivo histograma de tamanho de partícula; d) $100 \mathrm{~nm}$; e) $120 \mathrm{~nm}$; e f) $150 \mathrm{~nm}$.

Esta fórmula indica o ponto central no qual a distribuição da frequiência (área superficial ou volume/massa) deve girar. A vantagem em utilizar esse método está no fato de que a fórmula não requer o conhecimento do número de partículas analisadas, tal como na MET ${ }^{[26]}$.

A Tabela 4 compara as medidas de tamanho de partícula, obtidas pela técnica de MET (média das 3 espessuras) e pela LALLS para medir o $\mathrm{D}^{[4,3]}$.

Com os resultados obtidos pode-se confirmar que tanto a MET quanto a LALLS podem medir os tamanhos de partícula de borracha e sua distribuição, embora os valores de diâmetros médios $\mathrm{D}^{[4,3]}$ obtidos por LALLS sejam maiores do que aqueles obtidos por MET nas duas amostras analisadas. Os valores de $\mathrm{D}^{[4,3]}$ calculados para as amostras analisadas por MET também são maiores que o valor de $\mathrm{D}_{\mathrm{M}}$, ilustrando a influência das partículas maiores no valor do diâmetro médio calculado pelas duas técnicas. A largura da distribuição medida através do valor de $\mathrm{DD}_{\text {eq }}$ é consistente para os HIPS 1 e 2 , mostrando que quanto
Tabela 4. Valores de $\mathrm{D}^{[4,3]}$ medidos por ambas as técnicas.

\begin{tabular}{ccc}
\hline Material & \multicolumn{2}{c}{$\mathbf{D}^{[4,3]}(\mu \mathbf{m})$} \\
\cline { 2 - 3 } & MET & LALLS \\
\hline HIPS 1 & 1,4 & 2,3 \\
HIPS 2 & 2,9 & 2,9 \\
\hline
\end{tabular}

maior $\mathrm{DD}_{\text {eq }}$, maior a largura da distribuição do tamanho de partículas. Neste caso, os valores de $\mathrm{D}^{[4,3]}$ calculados para MET e LALLS divergiram conforme dados apresentados na Tabela 4. No entanto, uma equivalência entre os métodos foi observada para os valores de $\mathrm{D}^{[4,3]}$ obtidos para o HIPS 2. Portanto, a largura da distribuição $\mathrm{DD}_{\mathrm{eq}}$ pode ser um dos fatores que influenciam as diferenças nos valores obtidos para MET e LALLS.

\section{Conclusões}

De maneira geral, pode-se afirmar que a análise morfológica quantitativa por MET é uma poderosa técnica para caracterização de plásticos tenacificados. O método apresenta 
algumas vantagens em relação à análise por LALLS, por não utilizar solventes para dissolução preferencial da fase borracha, não há o risco de obter partículas inchadas além de permitir a avaliação das subinclusões nas partículas de borracha. Todavia, as micrografias do MET apresentam medidas bidimensionais que estão sujeitas a influência da espessura do filme e também da largura da distribuição dos tamanhos das partículas, conforme observado no presente trabalho. Além disso, a preparação da amostra para a MET é um processo trabalhoso e requer ataque químico das insaturações da borracha, para obter o contraste entre as fases.

A técnica permite a determinação dos parâmetros relacionados ao diâmetro médio de partículas e a sua distribuição, bem como a fração volumétrica aparente das partículas. Os métodos MET e LALLS apresentam resultados equivalentes para medidas de tamanho médio de partícula embora a largura da distribuição de tamanhos de partículas na amostra possa alterar essa equivalência entre os métodos. $\mathrm{Na}$ análise comparativa da microscopia com a técnica de LALLS observa-se que no HIPS 1, os valores obtidos por MET diferem significativamente daqueles obtidos por LALLS.

Para analisar o efeito da dispersividade do espectro de tamanho de partículas na equivalência entre os métodos, recomenda-se para trabalhos futuros o uso de um padrão monodisperso.

\section{Agradecimentos}

Os autores agradecem à Innova, S.A. pela bolsa de mestrado da aluna Juliana Rovere.

\section{Referências Bibliográficas}

1. Lovell, P. A. - "Rubber-Toughened Plastics: Strategies for their Preparation an Evaluation", http://academic. sun.ac.za/unesco/PolymerED2001/Contributions/LecUNESCO/Lovell.pdf, consultado em 18 de Abril de 2007.

2. Dear, J. P. - J. Mat. Sci., 38, p.891 (2003).

3. Bucknall, C. B. - "Toughened Plastics", Applied Science Publishers, London (1977).

4. Lee, S. J.; Jeoung, H. G.; \& Ahn, K. H. - J. Appl. Polym. Sci., 89, p.3672 (2003).

5. Grassi, V. G.; Forte, M. M. C. \& Dal Pizzol, M. F. - Polímeros: Ciência e Tecnologia, 11, 3, p.158 (2001).

6. Zhang, J.; Wang, X.; Lu, L.; Li, D. \& Yang, X. - J. Appl. Polym. Sci., 87, p.381 (2003).

7. Morales, G.; Leon, R. D.; Acuña, P.; Flores, R. F. \& Robles, Antonio M. - Polym. Eng. Sci., 46, p.1333 (2006).

8. Aiamsen, P.; Paiphansiri, U.; Sangribsub, S.; Polpanich, D. \& Tangboriboonrat, P. - Polym. Int., 52, p.1198 (2003).
9. Echte, A. - "Rubber-Toughened Plastics", C. K. Riew, ACS, Washington, D. C. (1989).

10. Dal Pizzol, M. F. - "Desenvolvimento de Poliestireno de Alto Impacto com Balanço Otimizado de Propriedades Mecânicas", Tese de Doutorado, Universidade Federal do Rio Grande do Sul, Brasil (2005).

11. Rivera, M. R.; Herrera, R \& Rios, L. - J. Elast. Plast., 38, p.133 (2006).

12. Ruffing, N. R. et al. - Process for making graft copolymers of vinyl aromatics compounds and stereospecific rubbers, Patente Americana, 3, 243, 481, 1966.

13. Grassi, V. G. - "Estudo da Resistência Química de Poliestireno de Alto Impacto às Gorduras", Dissertação, Universidade Federal do Rio Grande do Sul, Brasil (2002).

14. Mello, I.; Coutinho, F. \& Soares, B. - Química Nova, 27, 2, p.277 (2004).

15. Lacoste, J.; Delor, F.; Pilichowski, J. F.; Singh, R. P.; Prasad, A. V. \& Sivaram, S. - J. Appl. Polym. Sci., 59, p.953 (1996).

16. Silas, R. S.; Yates, J. \& Thornton, V. - Determination of Unsaturation Distribution in Polybutadienes by Infrared Spectrometry, Analytical Chemistry, 31, 4, p.529, 1959.

17. Mello I. L.; Soares, B. G.; Coutinho, F. M. B. \& Nunes, D. S. S. - Polímeros: Ciência e Tecnologia, 17, 1, p.62 (2007).

18. Pires, N. M. T.; Lira, C. H.; Ferreira, A. A.; Coutinho, P. L. \& Nicolini, P. L. - "Desempenho de Polibutadienos com Alto Teor de Cis" in: Anais do $9^{\circ}$ Congresso Brasileiro de Tecnologia da Borracha, p.1, São Paulo-SP, novembro (2001).

19. Canto, L. B.; Mantovani, G. L.; Azevedo, E. R.; Bonagamba, T. J.; Hage, E. \& Pessan, L. A. - Polym. Bulletin, 57, p.513 (2006).

20. Bayer. Brochura Técnica, 1 (2003), citada em Dal Pizzol, M. F. - "Desenvolvimento de Poliestireno de Alto Impacto com Balanço Otimizado de Propriedades Mecânicas", Tese de Doutorado, Universidade Federal do Rio Grande do Sul, Brasil (2005).

21. Katime, I.; Quintana, J. R. \& Price, C. - Mat. Lett., 22, p.297 (1995).

22. Innova, Boletim Técnico, (1998), citado Grassi, V. G.; Forte, M. M. C. \& Dal Pizzol, M. F. - Polímeros: Ciência e Tecnologia, 11, 3, p.158 (2001).

23. Correa, C. A. - "Microscopia Eletrônica de Transmissão”, in: Técnicas de Caracterização de Polímeros, cap. 10, S. V. Canevarolo Jr, Ed. Artliber, São Paulo (2003).

24. Correa, C. A. - Polímeros: Ciência e Tecnologia, Jan./ Mar, p.24 (1995). 
25. Pires, P. A. R. \& El Seoud, O. A. - "Espalhamento de Luz”, in Técnicas de Caracterização de Polímeros, cap. 4, S. V. Canevarolo Jr, Ed. Artliber, São Paulo (2003).

26. Hall, R. A.; Hites, R. D. \& Plantz, P. - J. Appl. Polym. Sci., 27, p.2885 (1982).

27. Rawle, A. - "Basic Principles of Particle Size Analysis”, Technical Paper, Malvern Instruments Limited, Worcestershire, Reino Unido, p.1 (2000).
28. Correa, C. A.; Yamakawa, R. S.; Horiuchi, L. N. \& Canevarolo, S. V. - "Proposta de uma Metodologia para a Avaliação do Tamanho Médio de Partículas e sua Distribuição em Plásticos Tenacificados com Elastômeros", in: Anais do $13^{\circ}$ Congresso Brasileiro de Engenharia e Ciência dos Materiais, p.4068, Curitiba-PR, dezembro (1998).

Enviado: 25/04/07 Reenviado: $20 / 06 / 07$ Aceito: $25 / 06 / 07$ 